\title{
Lässt sich der Fettleber bald Paroli bieten?
}

Eine effektive medikamentöse Therapie der nicht-alkoholischen Fettlebererkrankung (NAFLD) und der nicht-alkoholischen Steatohepatitis (NASH) gibt es bislang nicht. Allerdings sind einige vielversprechende Wirkstoffe in klinischer Entwicklung. Ein Hoffnungsträger ist vor allem die nor-Ursodesoxycholsäure (nor-UD-

(A), wie beim 23. Symposium „Aktuelle Hepatologie 2017: Hot Topics" in Mannheim deutlich wurde.

Bei der Behandlung chronischer Lebererkrankungen zeichnen sich laut Prof. Michael Manns, Hannover, deutliche Fortschritte ab. So dürften aus seiner Sicht, analog zur Situation bei der Hepatitis C, schon bald auch bei anderen chronischen
Lebererkrankungen neue Therapieoptionen verfügbar werden und nachhaltige Behandlungserfolge zu erzielen sein. Das gilt unter anderem für die NAFLD und für $\mathrm{NASH}$, deren Bedeutung lange unterschätzt wurde. Die Inzidenz und Prävalenz dieser Fettlebererkrankungen nimmt stetig zu. Bei einer Prävalenz von $20-30 \%$ weltweit handelt es sich nach Prof. Heike Bantel, Hannover, durchaus bereits um „eine neue Volksseuche“.

\section{Dringender Bedarf für neue Therapieoptionen}

Da bislang keine für diese Indikation zugelassenen Medikamente verfügbar sind, bleibt zunächst nur die Motivation des Patienten zur Gewichtsreduktion und zur vermehrten körperlichen Aktivität. Al- lerdings ist zur Besserung charakteristischer histologischer Befunde bei NASH eine Reduktion des Körpergewichts um 5-10\% erforderlich, ,ein Therapieziel, das nur von wenigen Patienten erreicht wird", so Bantel.

Es besteht damit ein dringender Bedarf für neue Therapieoptionen. Dabei sind derzeit gleich mehrere Wirkstoffe in klinischer Entwicklung. Hoffnungen setzen die Hepatologen vor allem auf Farnesoid-X-Rezeptor (FXR)-Agonisten wie die Obeticholsäure sowie auf den PPAR- $\alpha / \delta$-Agonisten Elafibranor und den PPAR $\gamma$-Agonisten Pioglitazon. Ein weiterer vielversprechender Wirkstoff ist nor-UDCA, die in präklinischen Studien antiinflammatorische und antifibrotische Effekte gezeigt hat.

\section{"Aufbruch in ein neues Zeitalter" \\ PSC: Hoffnung auf Therapiefortschritte}

Die Ätiologie der chronisch entzündlichen Gallenwegserkrankungen ist letztlich noch nicht geklärt, allerdings werden die molekularen Mechanismen des Gallensäuretransports und des Gallensäuresignallings inzwischen besser verstanden. Das bietet Chancen für die Entwicklung neuer Therapieoptionen.

Vor allem im Hinblick auf die Behandlungsmöglichkeiten cholestatischer Lebererkrankungen zeichnet sich nach Prof. Michael Trauner, Wien, „der Aufbruch in ein neues Zeitalter in der He- patologie“ ab. Relativ günstig ist die Situation derzeit bei der primär biliären Cholangitis (PBC), bei der Ursodesoxycholsäure (UDCA, z.B. Ursofalk ${ }^{\circledast}$ ) die Standardtherapie ist. Die Mehrzahl der Patienten spricht gut auf die Gallensäure an.

Bei Patienten, die nicht adäquat auf UDCA ansprechen, gibt es mittlerweile mit der Obeticholsäure eine effektive Therapieoption. Problematisch aber ist der unter dieser Medikation oftmals auftretende Juckreiz. Deshalb wird weiter an der Entwicklung neuer Therapieoptionen gearbeitet. Hoffnungen gründen sich

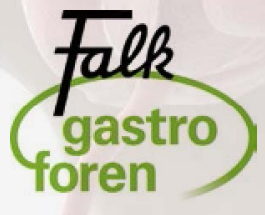

Besuchen Sie das nächste Falk Gastro Forum „Konservative und operative Therapien bei Erkrankungen von Leber und Ösophagus" am Samstag, den 28. Oktober 2017 in Dresden; Infos: www.falkfoundation.de/veranstaltungen nach Trauner auf PPAR-Liganden sowie auf Budesonid beim Overlapsyndrom der PBC und einer Autoimmunhepatitis und auch auf bereits verfügbare Biologika wie Ustekinumab und Rituximab.

\section{Signifikante Reduktion der Cholestase}

Auch bei der primär sklerosierenden Cholangitis (PSC) zeichnen sich Fortschritte ab. Sie werden vor allem vom Einsatz der nor-UDCA erwartet, einem Derivat der UDCA. Trauner präsentierte eine europaweite Phase-II-Studie, in der eine signifikante Reduktion der Cholestase unter norUDCA beobachtet wurde mit dosisabhängiger Besserung der Leberwerte bei zugleich sehr günstigem Sicherheitsprofil.

Christine Vetter

23. Symposium "Aktuelle Hepatologie 2017: Hot Topics" am 29. April 2017 in Mannheim, Veranstalter: Falk Foundation e.V. 